Supporting Information to:

\title{
Insights into the reaction mechanism of soluble epoxide hydrolase from theoretical active site mutants
}

\author{
Kathrin H. Hopmann and Fahmi Himo* \\ Department of Theoretical Chemistry, School of Biotechnology, Royal Institute of Technology, \\ AlbaNova University Center, SE-106 91 Stockholm, Sweden. \\ ${ }^{*}$ Corresponding author, email: himo@,theochem.kth.se
}

\section{Additional optimized geometries}

In all figures, asterisks indicate atoms that in calculations were kept fixed to their crystallographically observed positions. Schemes give detailed information about the structures and the optimized distances $(\AA)$. The substrate MSO corresponds to $(1 S, 2 S)$ - $\beta$-methylstyrene oxide, while SSO corresponds to $(S)$-styrene oxide.

Figure S1. Tyrosine single mutant model Y381F with MSO (attack at C1). (A) Reactant, (B) Covalent intermediate, (C) Tetrahedral intermediate (THI), (D) Product.

Figure S2. Tyrosine single mutant model Y465F with MSO (attack at C1). (A) Reactant, (B) Covalent intermediate, (C) TS for water attack, (D) Tetrahedral intermediate (THI), (E) TS for dissociation, (F) Product.

Figure S3. Tyrosine double mutant model Y381F/Y465F with MSO (attack at C1). (A) Reactant, (B) Covalent intermediate.

Figure S4. Alkylation transition states for attack at C2 of MSO in the tyrosine mutant models. (A) TS alkylation for C2 attack in the Y381 model, (B) TS alkylation for C2 attack in the Y465 model, (C) TS alkylation for C2 attack in the Y381/465 model. 
Figure S5. Tyrosine single mutant model Y381F with SSO. (A) Reactant, (B) TS alkylation for attack at $\mathrm{C} 1,(\mathrm{C}) \mathrm{TS}$ alkylation for attack at $\mathrm{C} 2$.

Figure S6. Tyrosine single mutant model Y465F with SSO. (A) Reactant, (B) TS alkylation for attack at $\mathrm{C} 1,(\mathbf{C}) \mathrm{TS}$ alkylation for attack at $\mathrm{C} 2$.

Figure S7. Tyrosine double mutant model Y381F/Y465F with SSO. (A) Reactant, (B) TS alkylation for attack at $\mathrm{C} 1,(\mathrm{C}) \mathrm{TS}$ alkylation for attack at $\mathrm{C} 2$.

Figure S8. His523N\&H model with MSO (attack at C1). A) Reactant, (B) Covalent intermediate.

Figure S9. His523NeH-H $\mathrm{H}_{2} \mathrm{O}$ model with MSO (attack at C1). A) Reactant, (B) Covalent intermediate.

Figure S10. NoHis523 model with MSO (attack at C1). A) Reactant, (B) Covalent intermediate. 
Figure S1 A-C
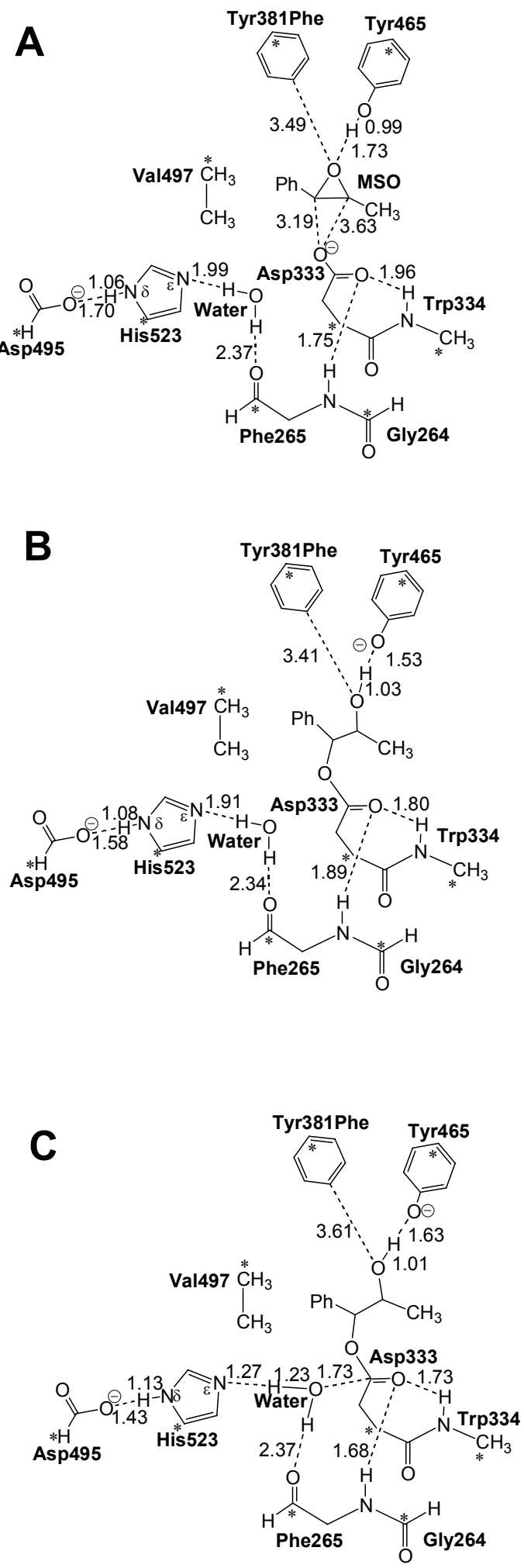
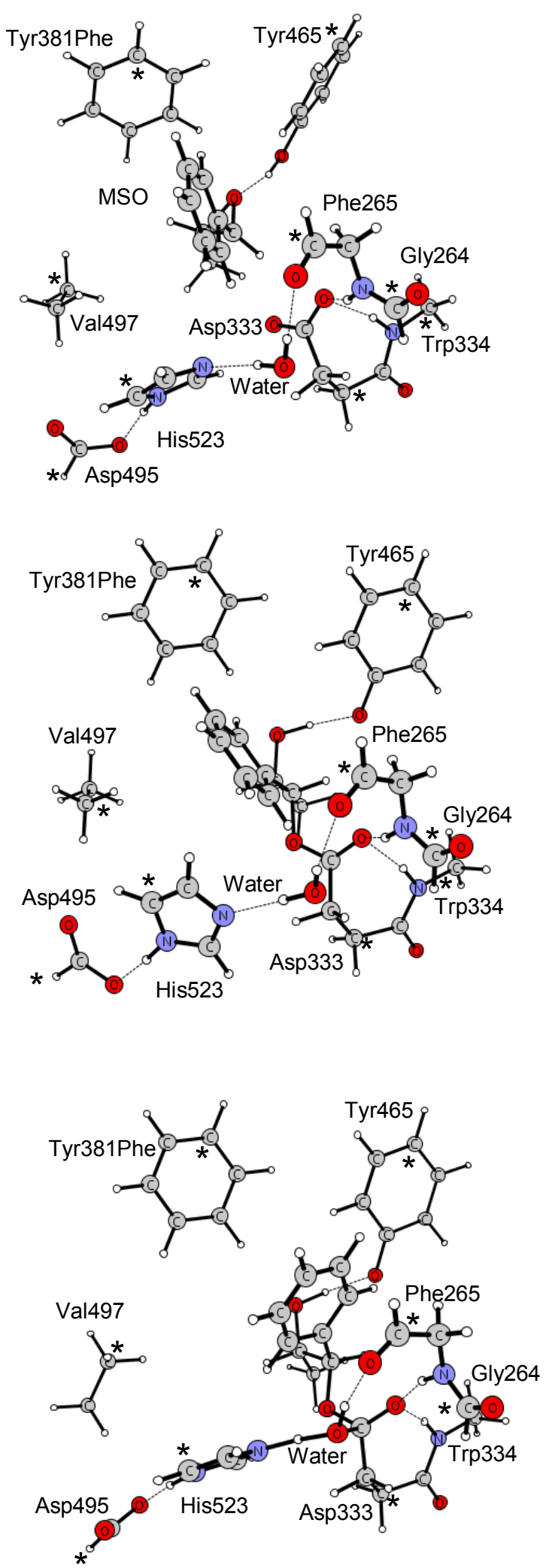
Figure S1 D-F
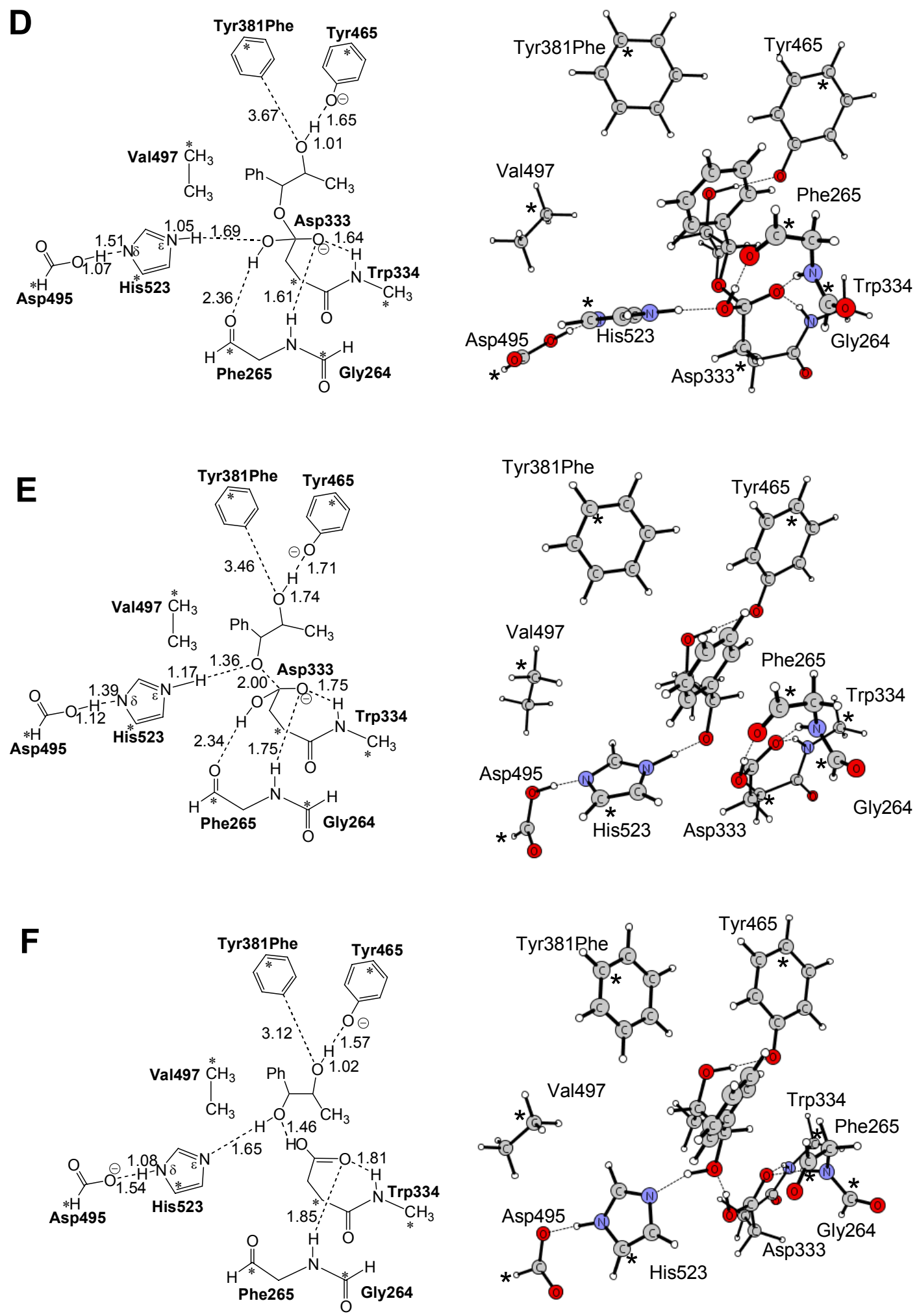


\section{Figure S2 A-C}
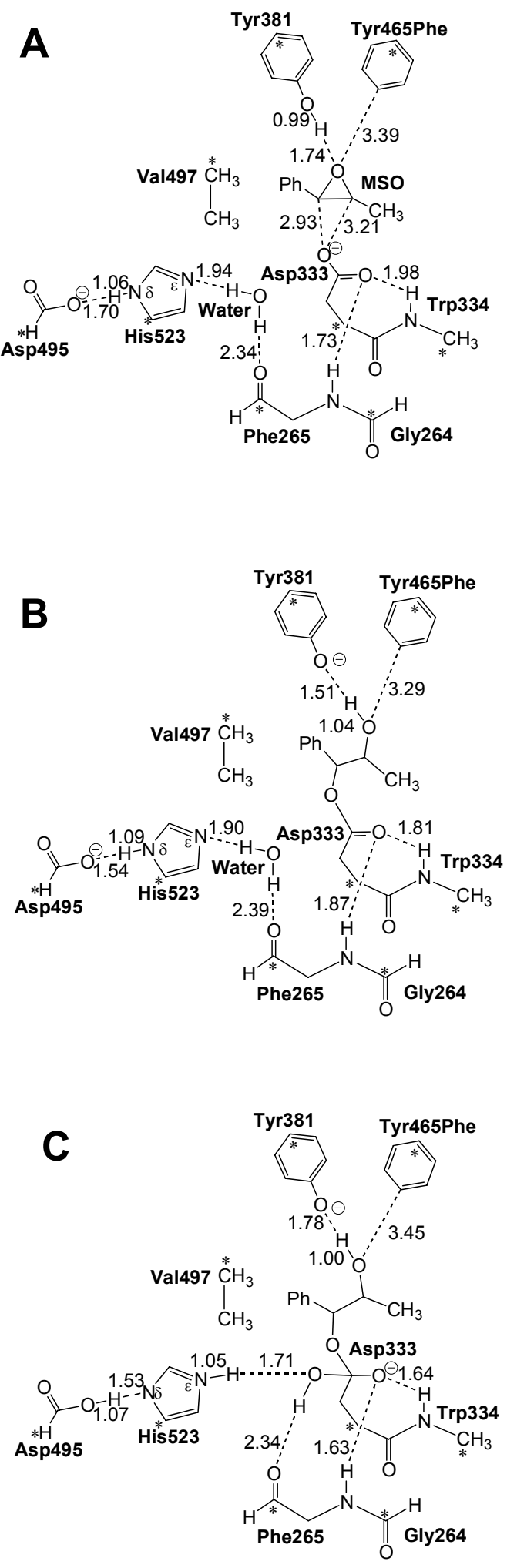
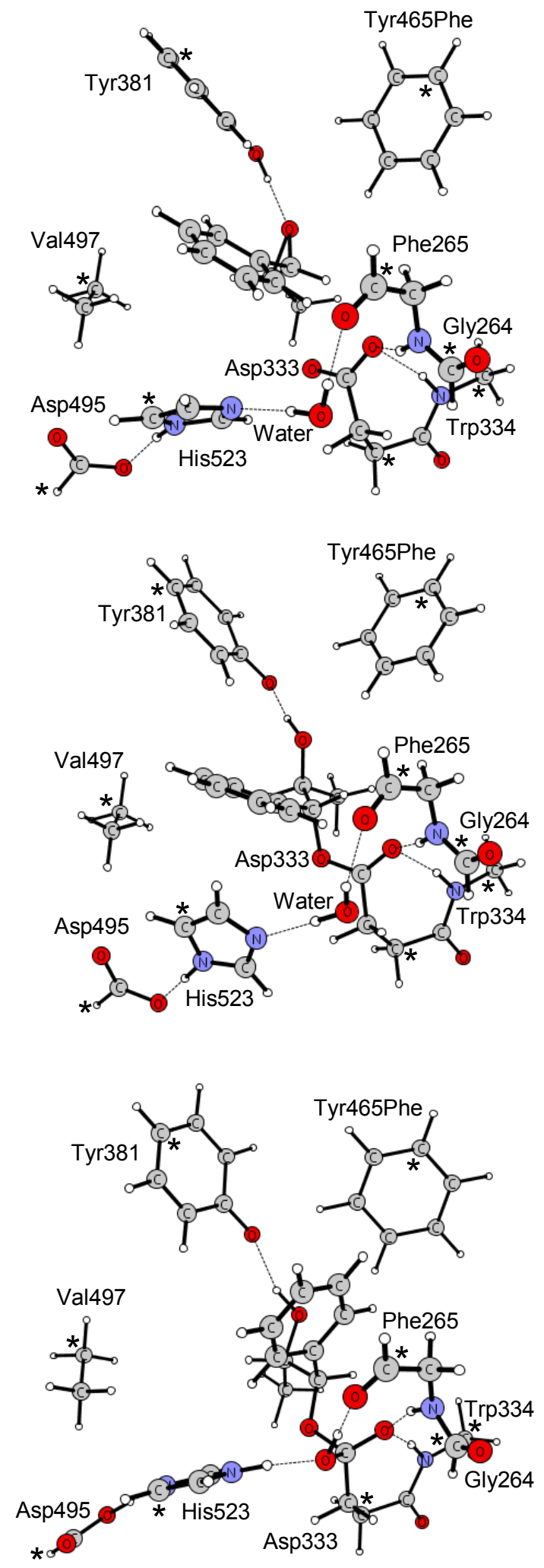
Figure S2D

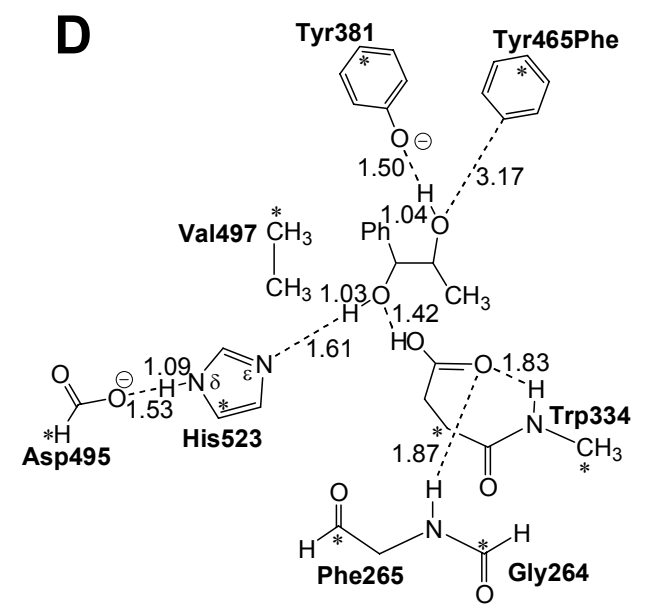

Figure S3 A-B
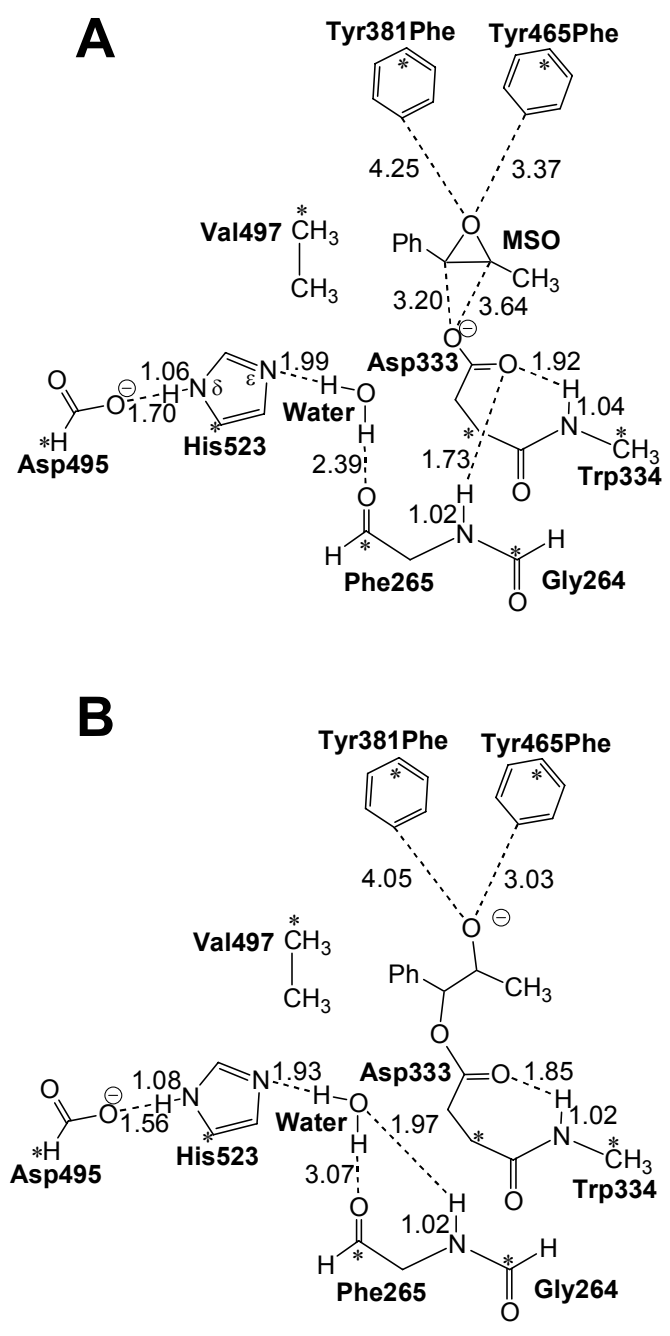

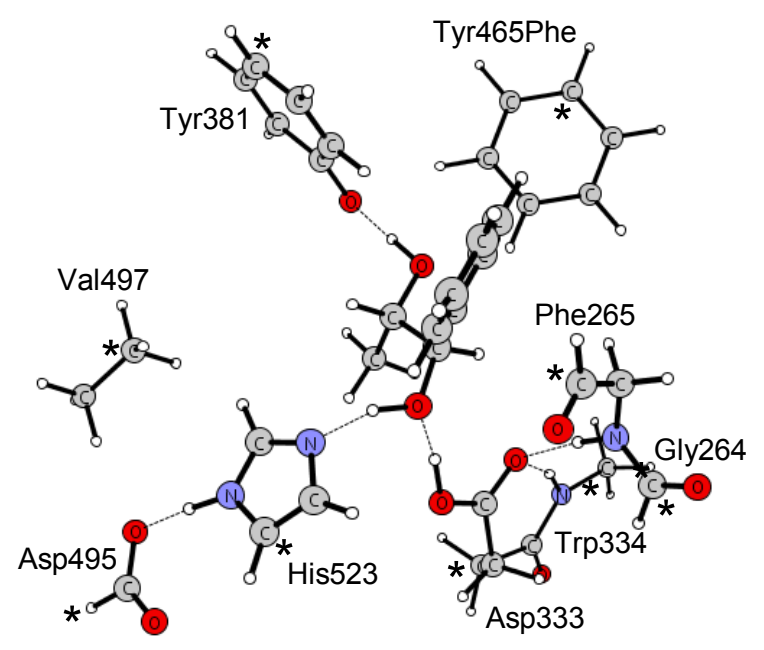

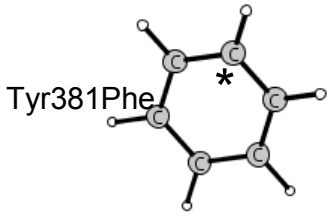

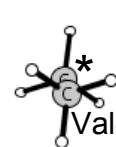

IVal497

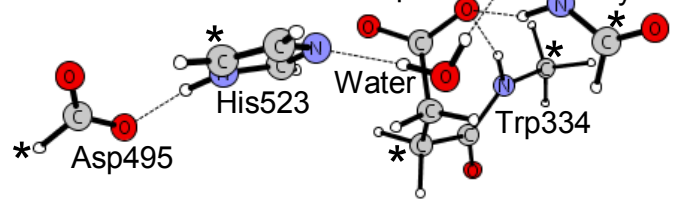

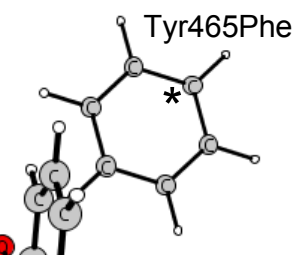<smiles>O[AsH2]</smiles>

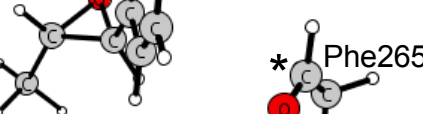

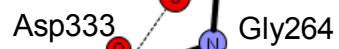

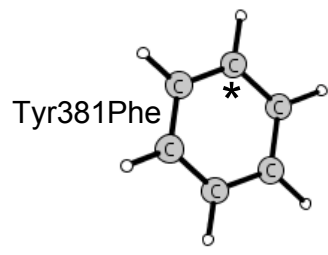

Tyr465Phe

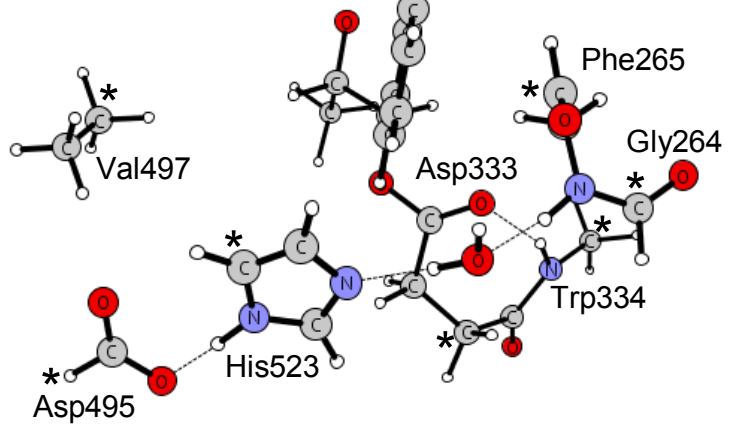


Figure S4 A-C

A

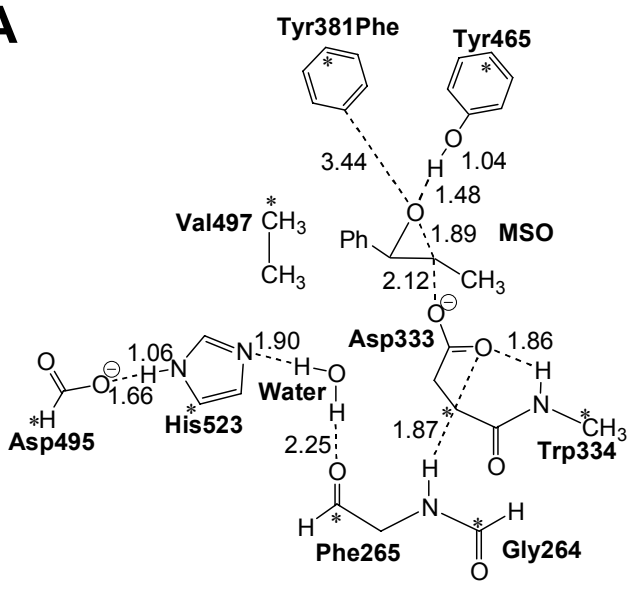

B

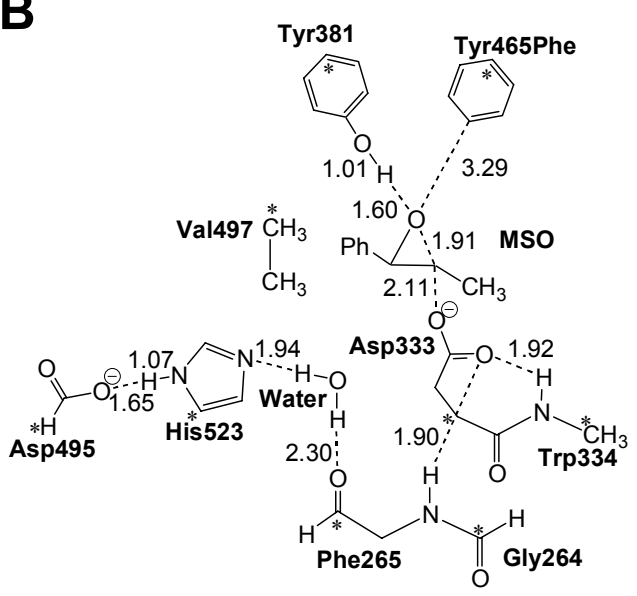

C
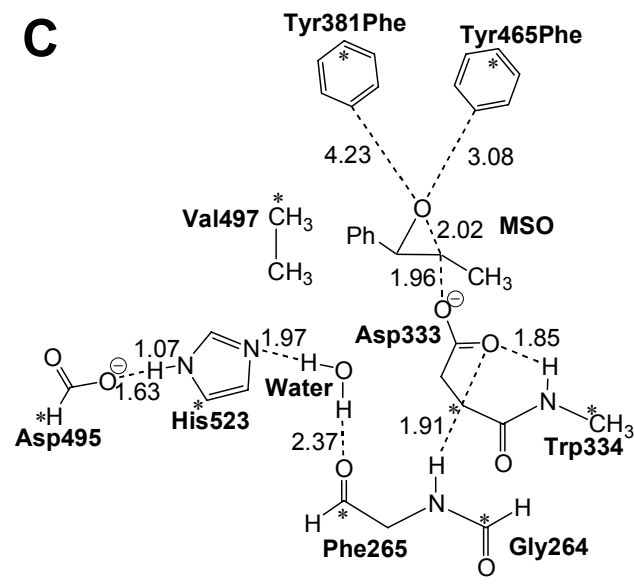
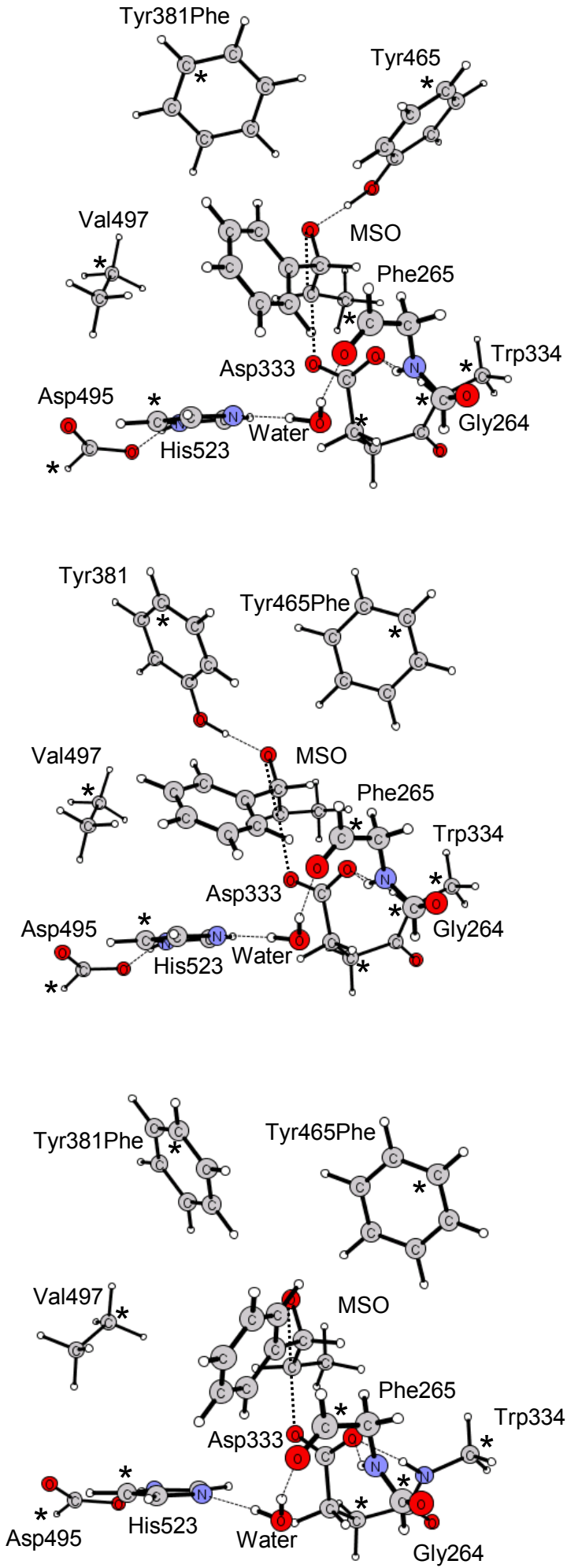
Figure S5 A-C
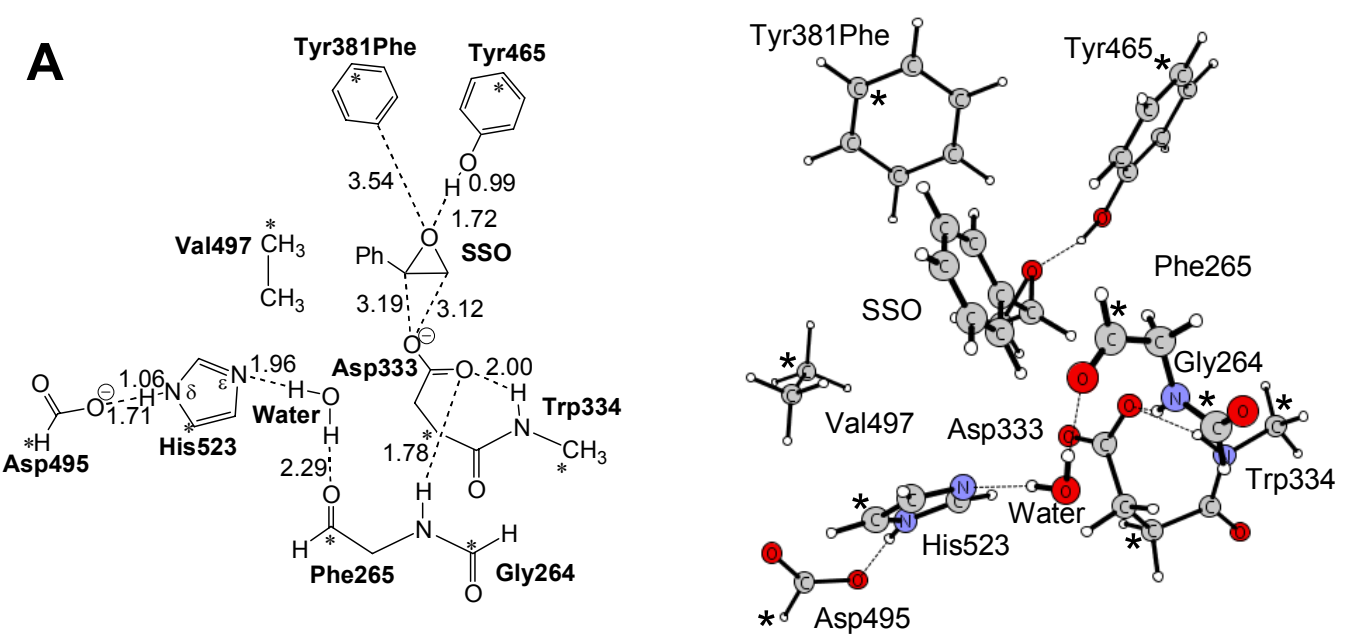

B
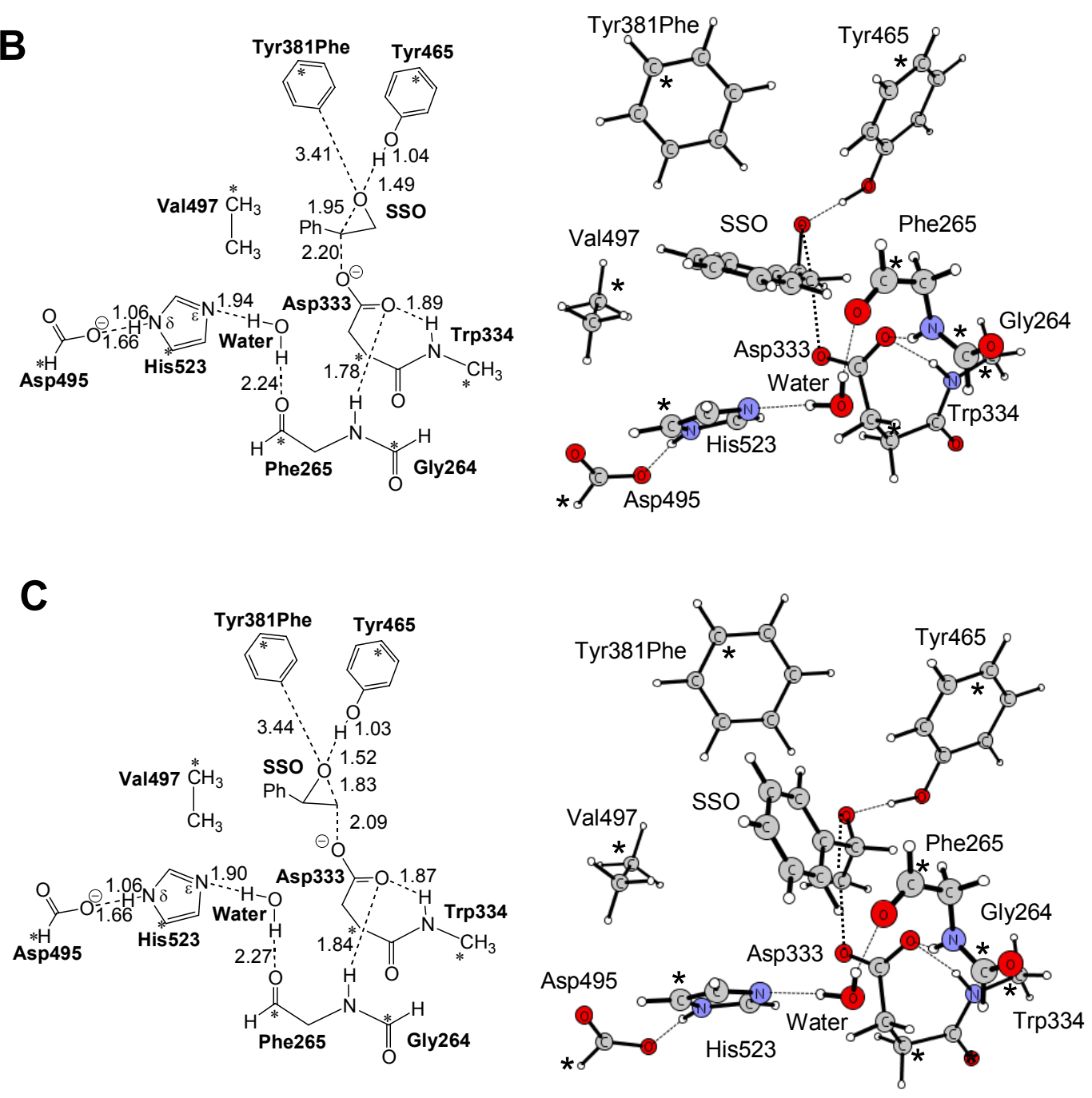


\section{Figure S6 A-C}
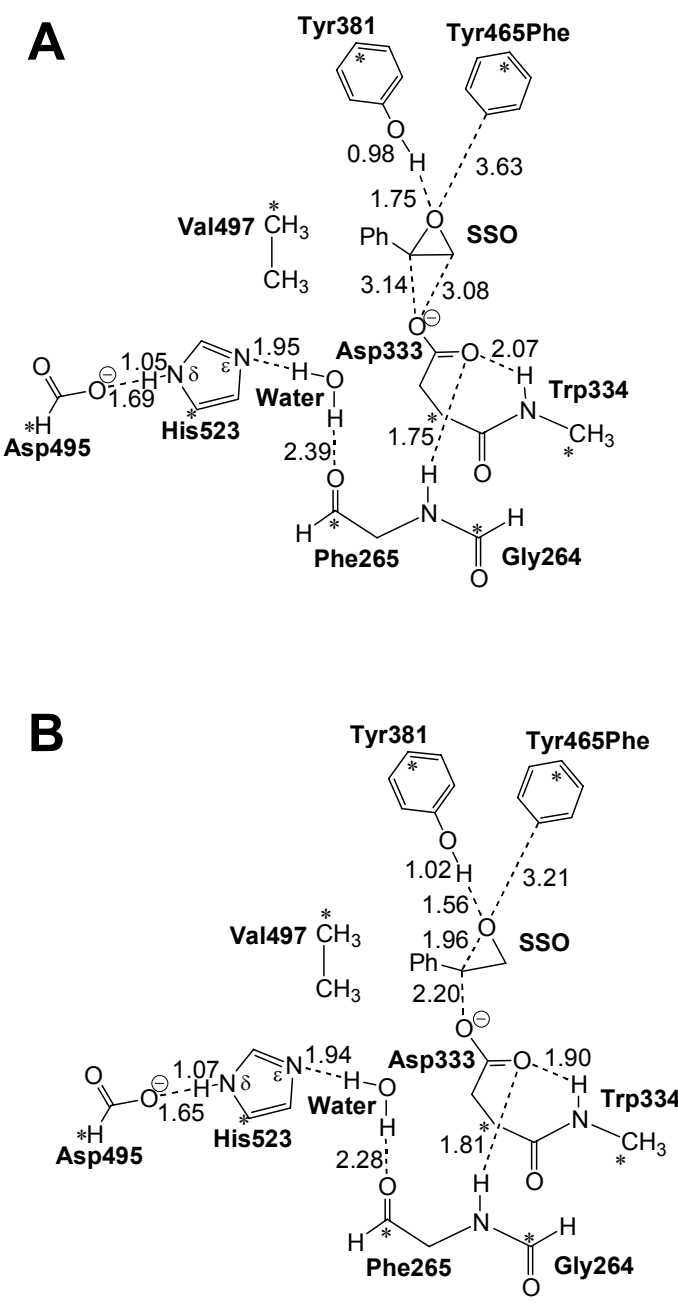

C
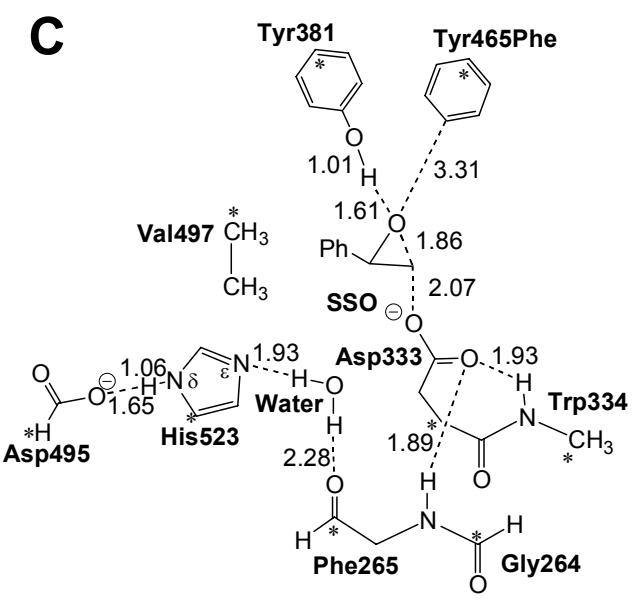
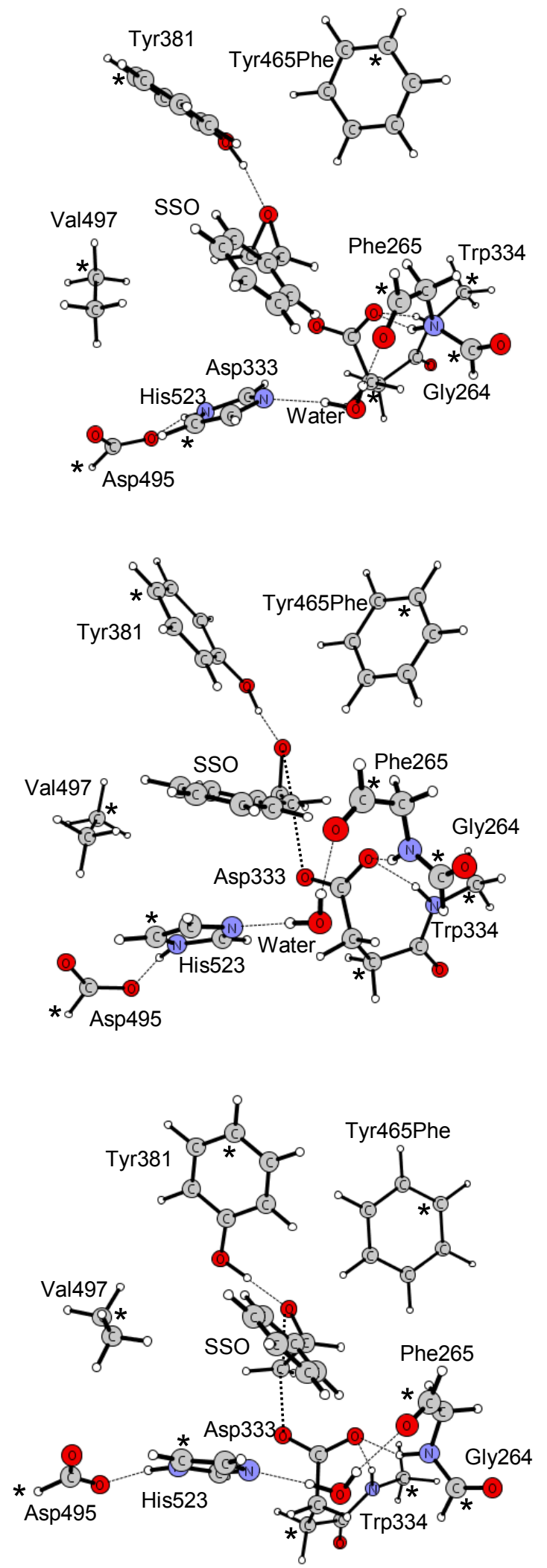
Figure S7 A-C
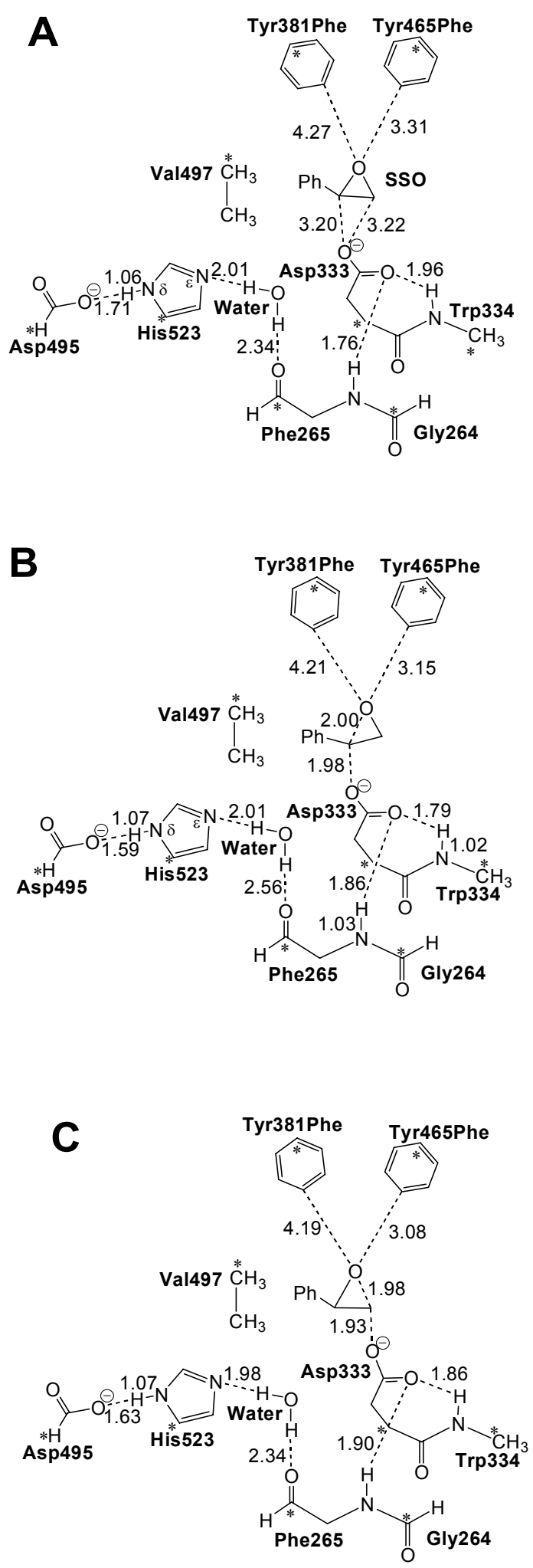
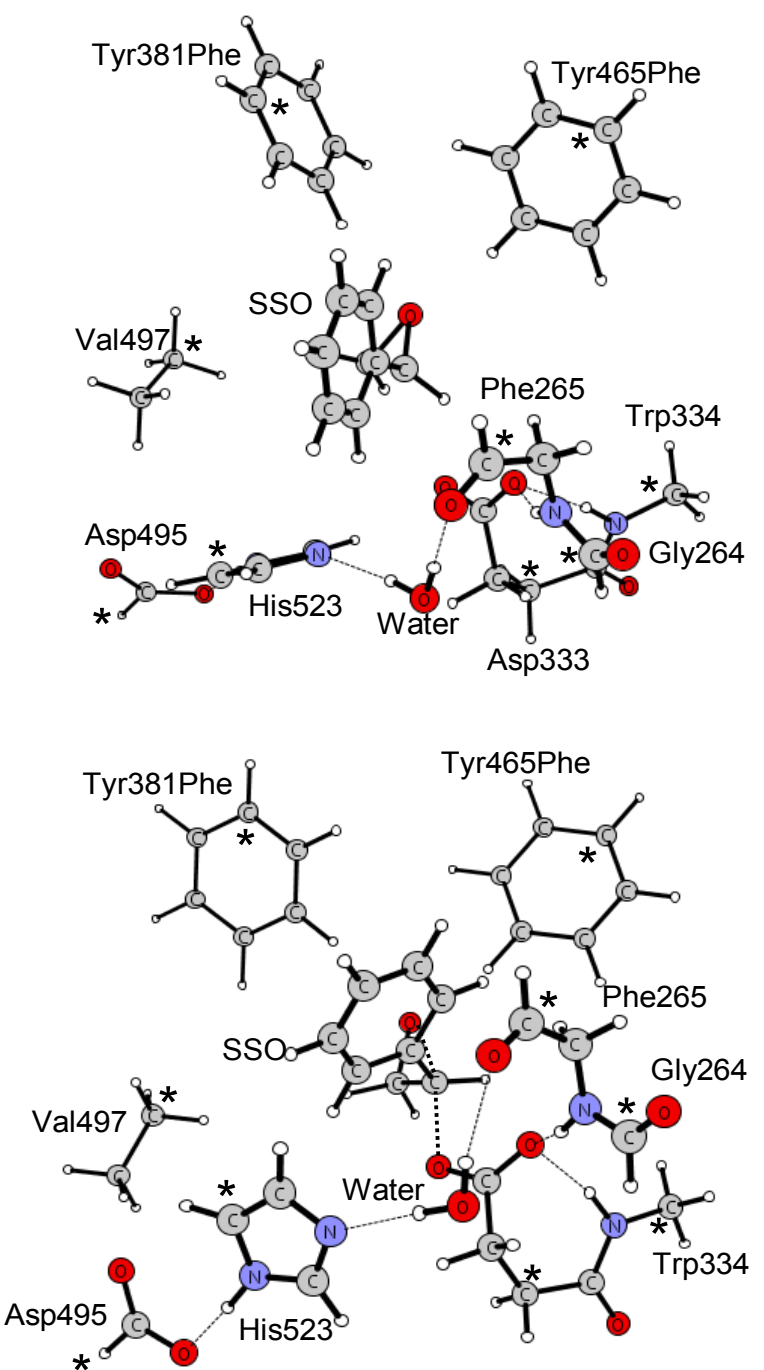

Asp333

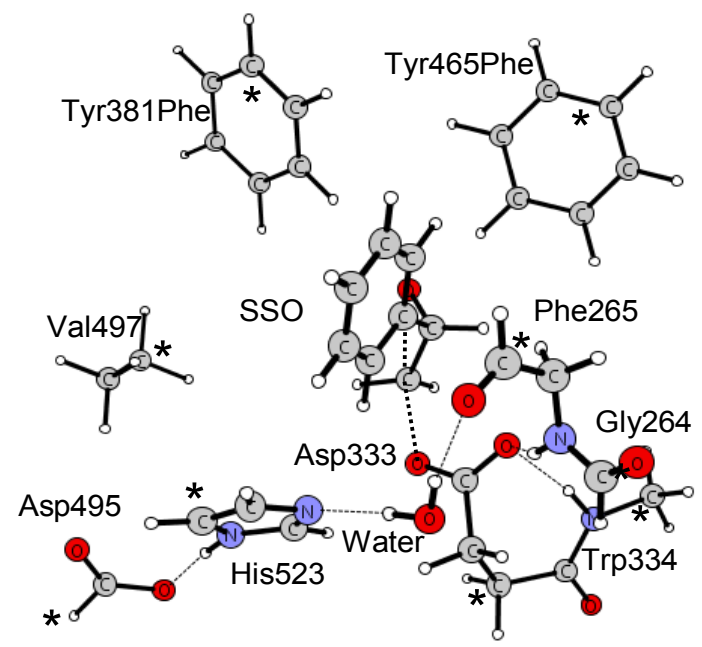


Figure S8 A-B

A

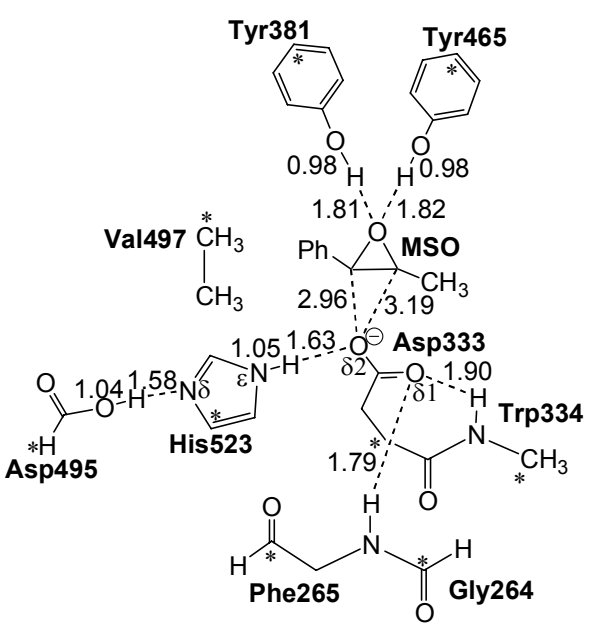

B

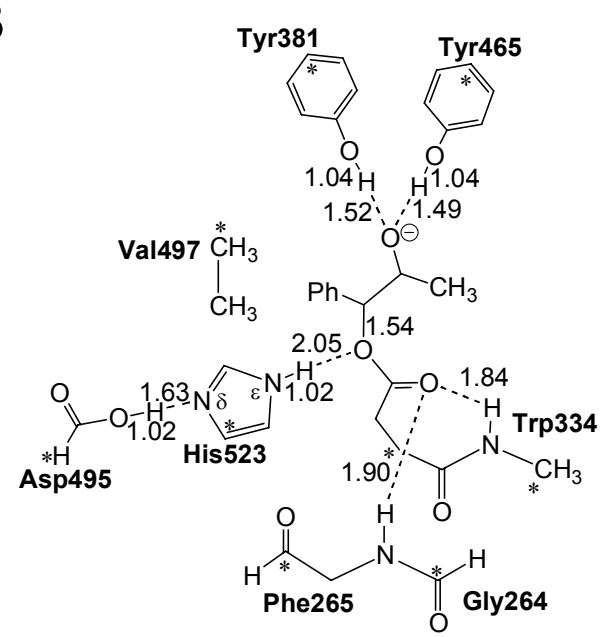

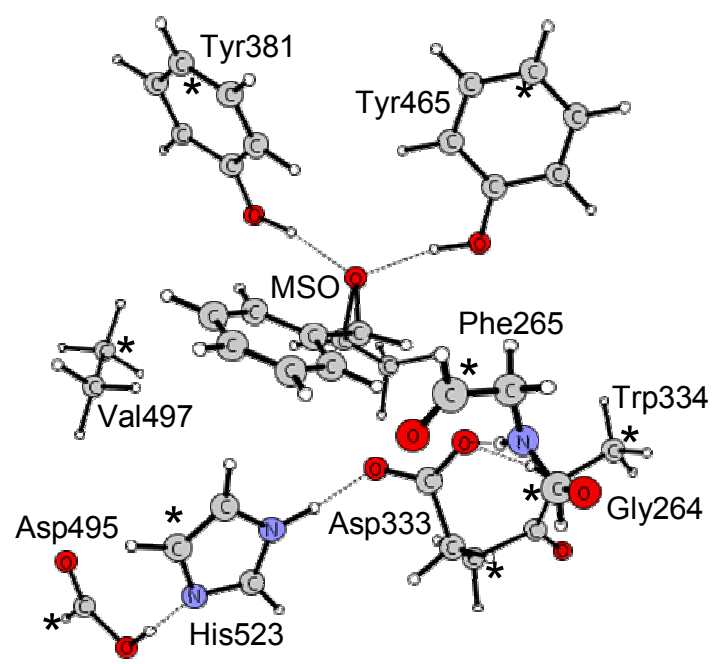

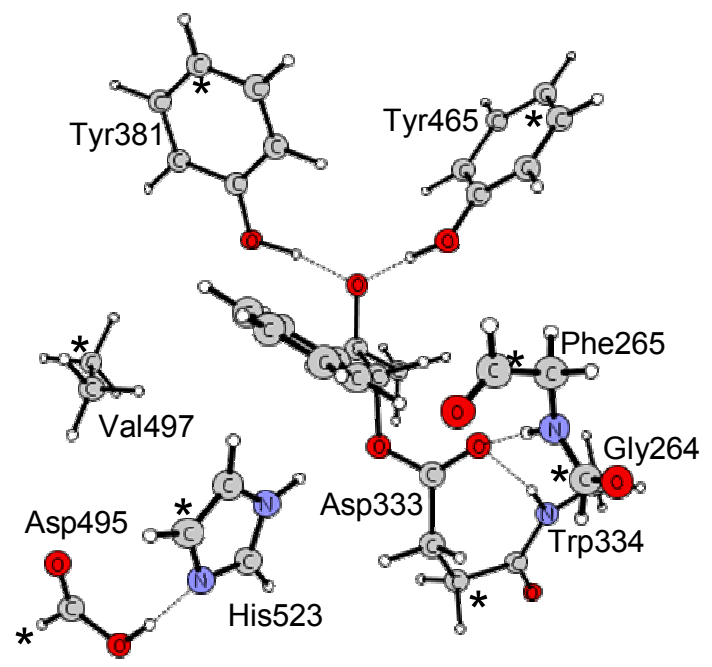


Figure S9 A-B

A
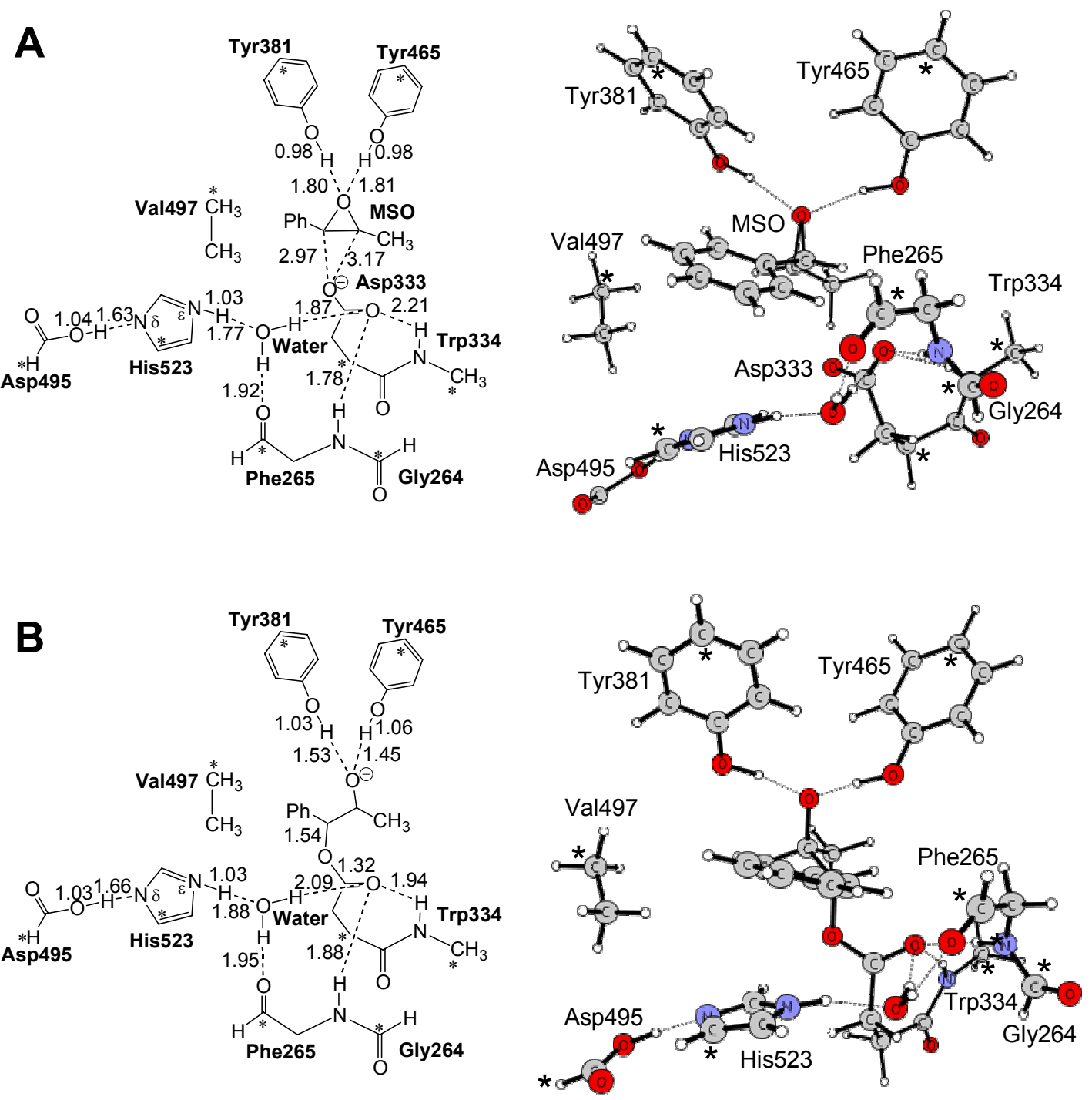
Figure S10 A-B
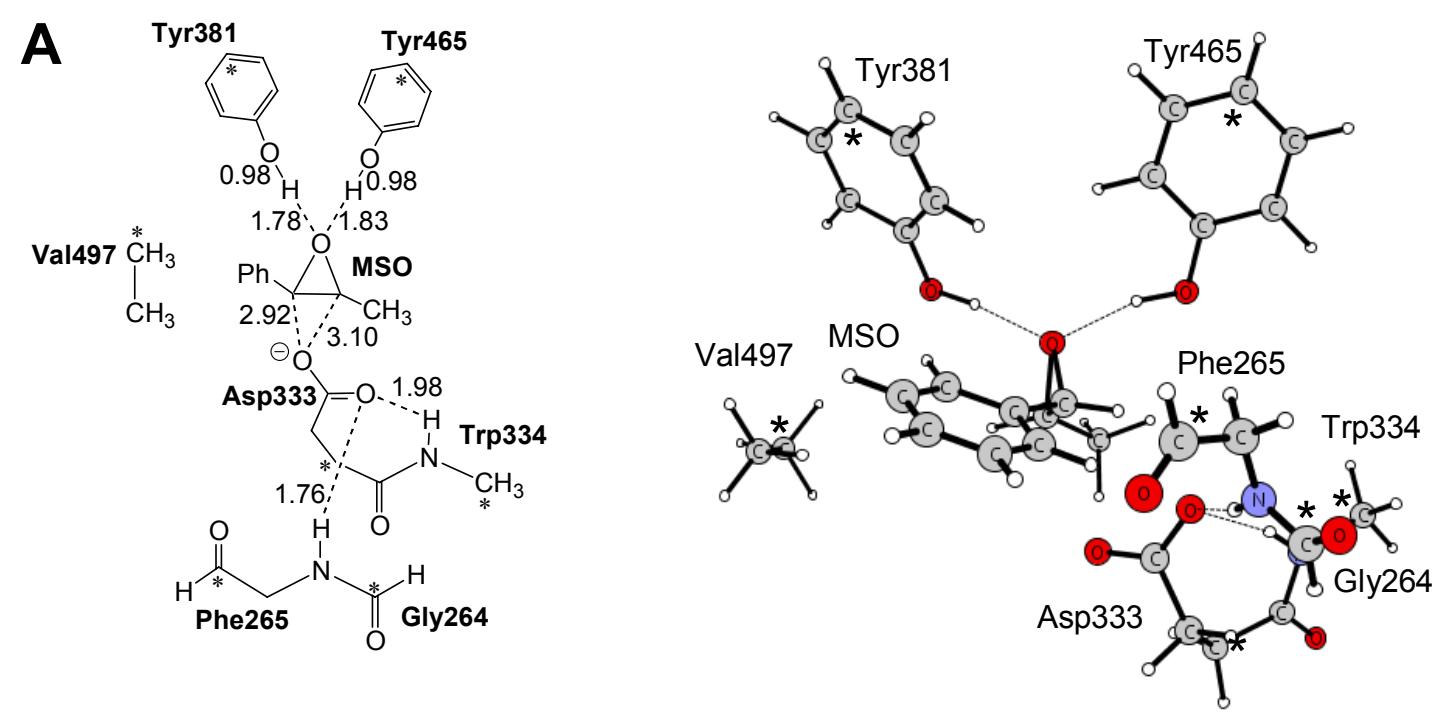

B
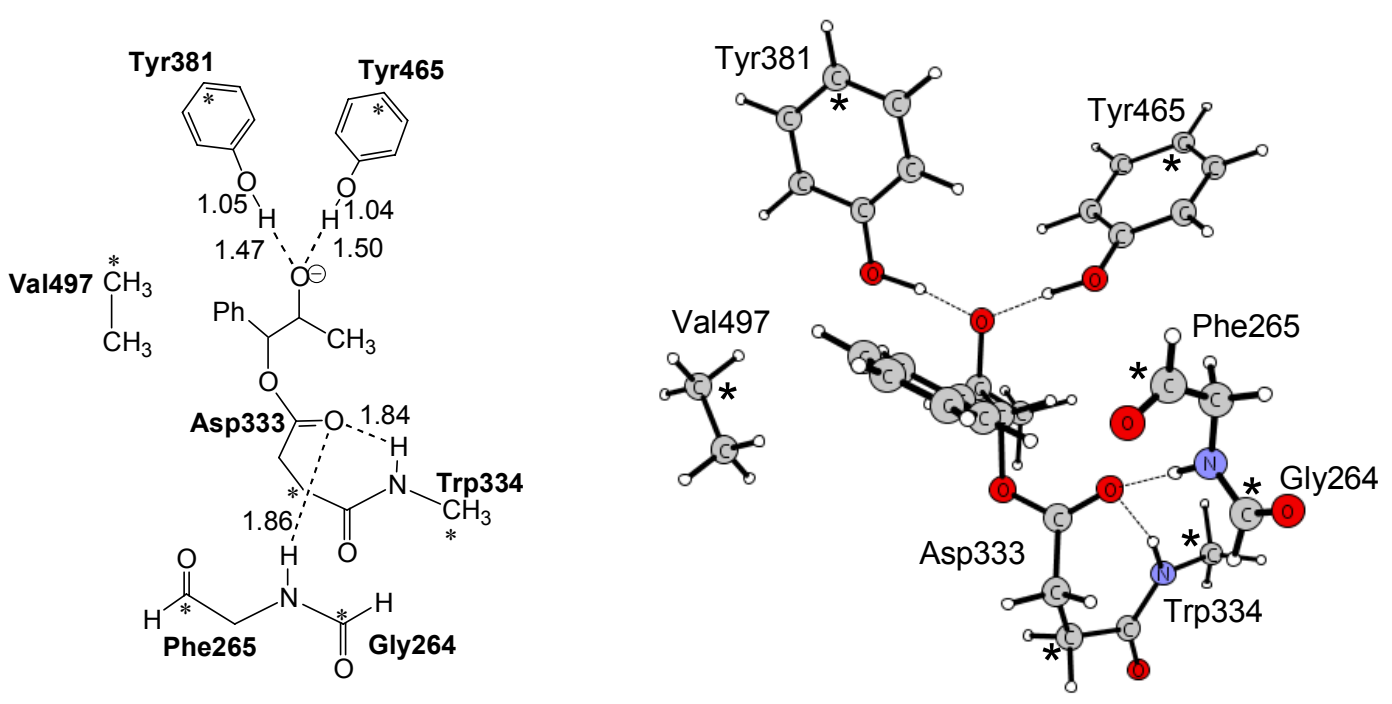
Table.S1 Computed absolute (a.u.) and relative energies (kcal/mol) including corrections terms for the basis set effect, solvation effect, and zero-point vibrational effect (ZPE).

\begin{tabular}{|c|c|c|c|c|c|c|c|c|}
\hline & \multicolumn{3}{|c|}{ Computed energies $^{\text {a }}$} & \multirow{2}{*}{$\begin{array}{c}\text { Relative } \\
\text { Energies }^{b} \\
6-31(\mathrm{dp})\end{array}$} & \multicolumn{3}{|c|}{ Energy corrections ${ }^{b}$} & \multirow{2}{*}{$\begin{array}{c}\text { Final } \\
\text { relative } \\
\text { energies }^{b}\end{array}$} \\
\hline & $6-31(d p)$ & $6-311+(2 d 2 p)$ & СРCM & & $\begin{array}{c}\text { Basis set } \\
\text { effect }\end{array}$ & $\begin{array}{l}t \text { CPCM } \\
\text { effect }\end{array}$ & $\begin{array}{l}\text { ZPV } \\
\text { effect }\end{array}$ & \\
\hline \multicolumn{9}{|c|}{ WT with MSO } \\
\hline Reactant & -2409.30131 & -2410.06023 & -2409.46130 & 0.0 & -476.2 & -100.4 & 498.0 & 0.0 \\
\hline Tsa-C1 & -2409.29172 & -2410.04992 & -2409.44839 & 6.0 & -475.8 & -98.3 & 497.3 & 7.8 \\
\hline CI-C1 & -2409.32627 & -2410.07965 & -2409.47856 & -15.7 & -472.8 & -95.6 & 497.4 & -8.0 \\
\hline prot. CI & -2409.32547 & -2410.08010 & -2409.47819 & -15.2 & -473.5 & -95.8 & 497.3 & -8.6 \\
\hline TSw-C1 & -2409.30613 & -2410.05290 & -2409.45465 & -3.0 & -468.6 & -93.2 & 495.7 & 9.5 \\
\hline THI-C1 & -2409.31621 & -2410.06075 & -2409.46360 & -9.4 & -467.2 & -92.5 & 498.9 & 8.5 \\
\hline TSd-C1 & -2409.30253 & -2410.04893 & -2409.44904 & -0.8 & -468.4 & -91.9 & 496.4 & 14.0 \\
\hline Prod-C1 & -2409.35179 & -2410.09961 & -2409.50110 & -31.7 & -469.3 & -93.7 & 498.3 & -17.7 \\
\hline TSa-C2 & -2409.28582 & -2410.04302 & -2409.44442 & 9.7 & -475.1 & -99.5 & 497.3 & 11.0 \\
\hline \multicolumn{9}{|c|}{ Y465F with MSO } \\
\hline Reactant & -2334.06834 & -2334.80032 & -2334.22708 & 0.0 & -459.3 & -99.6 & 494.9 & 0.0 \\
\hline TSa-C1 & -2334.04951 & -2334.78065 & -2334.20477 & 11.8 & -458.8 & -97.4 & 493.6 & 13.2 \\
\hline CI-C1 & -2334.07732 & -2334.80742 & -2334.23477 & -5.6 & -458.1 & -98.8 & 495.3 & -3.2 \\
\hline TSw-C1 & -2334.05964 & -2334.78158 & -2334.21186 & 5.5 & -453.0 & -95.5 & 492.5 & 13.4 \\
\hline THI-C1 & -2334.07192 & -2334.79115 & -2334.22233 & -2.2 & -451.3 & -94.4 & 495.9 & 11.9 \\
\hline TSd-C1 & -2334.05848 & -2334.77954 & -2334.20834 & 6.2 & -452.5 & -94.0 & 492.9 & 16.6 \\
\hline Prod-C1 & -2334.10488 & -2334.82697 & -2334.25767 & -22.9 & -453.1 & -95.9 & 495.4 & -12.5 \\
\hline TSa-C2 & -2334.04528 & -2334.77439 & -2334.20113 & 14.5 & -457.5 & -97.8 & 494.2 & 17.4 \\
\hline \multicolumn{9}{|c|}{ Y381F with MSO } \\
\hline Reactant & -2334.07283 & -2334.80502 & -2334.23366 & 0.0 & -459.5 & -100.9 & 494.6 & 0.0 \\
\hline TSa-C1 & -2334.05131 & -2334.78285 & -2334.20819 & 13.5 & -459.0 & -98.4 & 493.9 & 15.7 \\
\hline CI-C1 & -2334.08390 & -2334.81310 & -2334.23875 & -6.9 & -457.6 & -97.2 & 495.9 & $\mathbf{0 . 0}$ \\
\hline TSw-C1 & -2334.06577 & -2334.78626 & -2334.21782 & 4.4 & -452.1 & -95.4 & 493.3 & 16.1 \\
\hline THI-C1 & -2334.07484 & -2334.79301 & -2334.22716 & -1.3 & -450.7 & -95.6 & 496.4 & 14.7 \\
\hline TSd-C1 & -2334.06108 & -2334.78044 & -2334.21103 & 7.4 & -451.4 & -94.1 & 493.2 & 20.9 \\
\hline Prod-C1 & -2334.10532 & -2334.82860 & -2334.25574 & -20.4 & -453.9 & -94.4 & 496.3 & -6.5 \\
\hline TSa-C2 & -2334.04924 & -2334.77790 & -2334.20394 & 14.8 & -457.2 & -97.1 & 494.3 & 20.6 \\
\hline \multicolumn{9}{|c|}{ Y381F/Y465F with MSO } \\
\hline Reactant & -2258.83569 & -2259.54163 & -2258.99714 & 0.0 & -443.0 & -101.3 & 491.7 & 0.0 \\
\hline TSa-C1 & -2258.79939 & -2259.50716 & -2258.95380 & 22.8 & -444.1 & -96.9 & 490.4 & 24.8 \\
\hline CI-C1 & -2258.82725 & -2259.53089 & -2258.97874 & 5.3 & -441.5 & -95.1 & 491.7 & 13.1 \\
\hline TSa-C2 & -2258.79774 & -2259.50300 & -2258.95256 & 23.8 & -442.6 & -97.2 & 491.0 & 27.7 \\
\hline
\end{tabular}




\section{WT with SSO}

\begin{tabular}{l|lll} 
Reactant & -2369.97413 & -2370.72480 & -2370.13384 \\
TSa-C1 & -2369.96648 & -2370.71708 & -2370.12269 \\
TSa-C2 & -2369.96486 & -2370.71479 & -2370.12242
\end{tabular} \mid

Y381F with SSO

\begin{tabular}{l|lll|} 
Reactant & -2294.74667 & -2295.46990 & -2294.90803 \\
TSa-C1 & -2294.72722 & -2295.45088 & -2294.88439 \\
TSa-C2 & -2294.73043 & -2295.45116 & -2294.88529
\end{tabular}

Y465 with SSO

Reactant

TSa-C1 $-2294.74224$ $-2294.72464$

$-2295.46610$ $-2295.44758$

$-2294.90377$ $-2294.88050$

$\mathrm{TSa}-\mathrm{C} 2$

$-2295.44633 \quad-2294.88131$

\begin{tabular}{l|lll|l}
0.0 & -471.1 & -100.2 & 480.6 & $\mathbf{0 . 0}$ \\
4.8 & -471.0 & -98.0 & 479.8 & $\mathbf{6 . 3}$ \\
5.8 & -470.6 & -98.9 & 479.9 & $\mathbf{7 . 0}$
\end{tabular}

\section{DobPhe with SSO}

\begin{tabular}{l|ccc|c|ccc|c} 
Reactant & -2219.51003 & -2220.20763 & -2219.67335 & 0.0 & -437.8 & -102.5 & 474.0 & $\mathbf{0 . 0}$ \\
TSa-C1 & -2219.47429 & -2220.17380 & -2219.63102 & 22.4 & -438.9 & -98.3 & 472.9 & $\mathbf{2 4 . 2}$ \\
TSa-C2 & -2219.47811 & -2220.17564 & -2219.63508 & 20.0 & -437.7 & -98.5 & 473.6 & $\mathbf{2 3 . 6}$
\end{tabular}

H523NeH with MSO

\begin{tabular}{l|ccc|c|ccc|c} 
Reactant & -2333.49192 & -2334.19598 & -2333.55012 & 0.0 & -441.8 & -36.5 & 491.1 & $\mathbf{0 . 0}$ \\
TSa-C1 & -2333.46894 & -2334.17185 & -2333.52775 & 14.4 & -441.1 & -36.9 & 490.6 & $\mathbf{1 4 . 2}$ \\
CI-C1 & -2333.47865 & -2334.18110 & -2333.54132 & 8.3 & -440.8 & -39.3 & 491.0 & $\mathbf{6 . 5}$
\end{tabular}

\section{H523NeH-H ${ }_{2} \mathrm{O}$ with MSO}

\begin{tabular}{l|ccc|c|ccc|c} 
Reactant & -2409.92648 & -2410.66651 & -2409.99020 & 0.0 & -464.4 & -40.0 & 507.9 & $\mathbf{0 . 0}$ \\
TSa-C1 & -2409.91045 & -2410.64520 & -2409.96923 & 10.1 & -461.1 & -36.9 & 506.7 & $\mathbf{1 5 . 3}$ \\
CI-C1 & -2409.92055 & -2410.65494 & -2409.98348 & 3.7 & -460.8 & -39.5 & 506.8 & $\mathbf{6 . 7}$
\end{tabular}

\section{NoHis523 with MSO}

\begin{tabular}{l|ccc|c|ccc|c} 
Reactant & -1917.45507 & -1918.03121 & -1917.51396 & 0.0 & -37.0 & -361.5 & 423.7 & $\mathbf{0}$ \\
TSa-C1 & -1917.44295 & -1918.01916 & -1917.49932 & 7.6 & -35.4 & -361.6 & 422.7 & $\mathbf{8 . 1}$ \\
CI-C1 & -1917.46295 & -1918.03814 & -1917.52162 & -4.9 & -36.8 & -360.9 & 422.9 & $\mathbf{- 4 . 9}$ \\
\hline
\end{tabular}

${ }^{\mathrm{a}}$ In a.u. ${ }^{\mathrm{b}}$ In $\mathrm{kcal} / \mathrm{mol}$ 